\title{
On Hidden Conflicts of Belief Functions
}

\author{
Milan DANIEL ${ }^{a}$ and Václav KRATOCHVÍL ${ }^{b}$ \\ ${ }^{a}$ Jan Becher - Karlovarská Becherovka, a.s., Pernod Ricard group, \\ Přemyslovská 43, CZ - 13000 Prague 3, Czech Republic, milan.daniel@pernod-ricard.com \\ ${ }^{b}$ Institute of Information Theory and Automation, Czech Academy of Sciences, \\ Pod Vodárenskou veží 4, CZ - 18208 Prague 8, Czech Republic, velorex@utia.cas.cz
}

\begin{abstract}
Hidden conflict of belief functions in the case where the sum of all multiples of conflicting belief masses being equal to zero was observed. Degrees of hidden conflict are defined and analyzed including their relation to partial and full non-conflictness. The hidden conflict between two belief functions is distinguished from internal hidden conflict(s) of the individual belief function(s). Finally, computational issues of hidden conflict and non-conflictness are presented.
\end{abstract}

Keywords: Belief functions, DempsterShafer theory, Uncertainty, Conflicting belief masses, Internal conflict, Conflict between belief functions, Hidden conflict, Full nonconflictness.

\section{Introduction}

When combining belief functions (BFs) by the conjunctive rules of combination, some conflicts often appear (they are assigned either to $\emptyset$ by non-normalised conjunctive rule (a) or distributed among other belief masses by normalization in Dempster's rule of combination $\oplus$ ). Combination of conflicting BFs and interpretation of their conflicts are often questionable in real applications.

Sum of all multiples of conflicting belief masses (denoted by $\left.m_{\circledast}(\emptyset)\right)$ was interpreted as a conflict between BFs in the classic Shafer's approach [20]. Nevertheless already in '90s examples of non-conflicting BFs with high $m_{\circledast}(\emptyset)$ were observed. Classification of high conflict is very important in the combination of BFs from different belief sources. Thus a series of papers related to conflicts of BFs was published, e.g. $[1,6,10,7,11,13,14,15,19,22]$.

A new interpretation of conflicts of belief functions was introduced in [4]: an important distinction of internal conflicts of individual BFs (due to their inconsistency) from conflicts between BFs (due to conflict/contradiction of evidence represented by the BFs) was introduced there. Note that zero-sum of all multiples of conflicting belief masses is usually considered as non-conflictness of the belief functions in all the mentioned approaches.

On the other hand, when analyzing the conflict between belief functions based on their non-conflicting parts $^{1}[7]$ a positive value of conflict was observed even in a situation when the sum of all multiples of conflicting belief masses equals to zero. The observed conflicts are not high, but they are against the generally accepted important classification of BFs to be mutually conflicting or mutually non-conflicting. Hence an analysis of this topic is required.

This arose a series of new questions: how to interpret the sum of conflicting masses $m_{\curvearrowleft}(\emptyset)$ ? Is the conflict based on non-conflicting parts of belief functions correct? Some of the answers are provided in this text. They are positive in favor of the conflict based on nonconflicting parts. This led us to a definition of a hidden conflict of BFs (Section 3).

Going further, different levels/degrees of hidden conflicts are defined and a maximal degree of hidden conflict is investigated. Analogously to the degrees of hidden conflict, there also exist different degrees of nonconflictness. Full non-conflictness and conditions, under which belief functions are fully non-conflicting, are defined and presented here; particular degrees of nonconflictness are just under investigation.

In accordance with the approach from [4], there are observed and presented not only hidden conflicts between two belief functions but also internal hidden conflicts of individual BFs (Section 5). Finally, computational aspects of hidden conflict are presented in Section 6 .

\footnotetext{
${ }^{1}$ Conflicting and non-conflicting parts of belief functions originally come from [5].
} 


\section{Preliminaries}

We assume classic definitions of basic notions from theory of belief functions [20] on finite exhaustive frames of discernment $\Omega_{n}=\left\{\omega_{1}, \omega_{2}, \ldots, \omega_{n}\right\}$.

A basic belief assignment (bba) is a mapping $m$ : $\mathcal{P}(\Omega) \longrightarrow[0,1]$ such that $\sum_{A \subset \Omega} m(A)=1$; the values of the bba are called basic belief masses (bbm). $m(\emptyset)=0$ is usually assumed. $\mathcal{P}(\Omega)=\{X \mid X \subseteq \Omega\}$ is a power-set of $\Omega$. A belief function $(B F)$ is a mapping $\operatorname{Bel}: \mathcal{P}(\Omega) \longrightarrow[0,1], \operatorname{Bel}(A)=\sum_{\emptyset \neq X \subseteq A} m(X)$. A plausibility function $\mathrm{Pl}: \mathcal{P}(\Omega) \longrightarrow[0,1], P l(A)=$ $\sum_{\emptyset \neq A \cap X} m(X)$. Because there is a unique correspondence among $m$ and corresponding $\mathrm{Bel}$ and $\mathrm{Pl}$, we often speak about $m$ as of a belief function.

A focal element is a subset of the frame of discernment $X \subseteq \Omega$, such that $m(X)>0$. If all focal elements are singletons (i.e. one-element subsets of $\Omega$ ), then we speak about a Bayesian belief function (BBF); in fact, it is a probability distribution on $\Omega$. If there are only focal elements such that $|X|=1$ or $|X|=n$ we speak about quasi-Bayesian $B F(q B B F)$. In the case of $m(\Omega)=1$ we speak about vacuous $B F(\mathrm{VBF})$ and about a non-vacuous $B F$ otherwise. In the case of $m(X)=1$ for $X \subset \Omega$ we speak about categorical BF. If all focal elements have a non-empty intersection, we speak about a consistent $B F$; and if all of them are nested, about a consonant $B F$.

Dempster's (normalized conjunctive) rule of combination $\oplus:\left(m_{1} \oplus m_{2}\right)(A)=\sum_{X \cap Y=A} K m_{1}(X) m_{2}(Y)$ for $A \neq \emptyset$, where $K=\frac{1}{1-\kappa}, \kappa=\sum_{X \cap Y=\emptyset} m_{1}(X) m_{2}(Y)$, and $\left(m_{1} \oplus m_{2}\right)(\emptyset)=0$, see [20]. Putting $K=1$ and $\left(m_{1} @ m_{2}\right)(\emptyset)=\kappa=m_{\circledast}(\emptyset)$ we obtain the nonnormalized conjunctive rule of combination ()$^{2}$, see e. g. $[21]$.

Smets' pignistic probability is given by $\operatorname{Bet} P\left(\omega_{i}\right)=$ $\sum_{\omega_{i} \in X \subseteq \Omega} \frac{1}{|X|} \frac{m(X)}{1-m(\emptyset)}$, see e.g. [21]. Normalized plausibility of singletons ${ }^{2}$ of $\mathrm{Bel}$ is a probability distribution $P l_{-} P$ such that $P l_{-} P\left(\omega_{i}\right)=\frac{P l\left(\left\{\omega_{i}\right\}\right)}{\sum_{\omega \in \Omega} P l(\{\omega\})}[2,3]$.

A conflict of BFs Bel',Bel" based on their nonconflicting parts $\mathrm{Bel}_{0}^{\prime}, \mathrm{Bel}_{0}^{\prime \prime}$ is defined by the expression $\operatorname{Conf}\left(\mathrm{Bel}^{\prime}, \mathrm{Bel}^{\prime \prime}\right)=\left(m_{0}^{\prime} @ m_{0}^{\prime \prime}\right)(\emptyset)$, where nonconflicting part $\mathrm{Bel}_{0}$ (of a $\mathrm{BF} \mathrm{Bel}$ ) is unique consonant BF such that $P l_{-} P_{0}=P l_{-} P$ (normalized plausibility of singletons corresponding to $\mathrm{Bel}_{0}$ is the same as that corresponding to $\mathrm{Bel}$ ); $m_{0}$ is a bba related to $\mathrm{Bel}_{0}$. For an algorithm to compute $B e l_{0}$ see [7].

\footnotetext{
${ }^{2}$ Plausibility of singletons is called contour function by Shafer in [20], thus $P l_{-} P(B e l)$ is a normalization of contour function in fact.
}

\section{Conflicts of Belief Functions}

Original Shafer's definition of the conflict measure between two belief functions [20] is the following: $\kappa=$ $\sum_{X \cap Y=\emptyset} m_{1}(X) m_{2}(Y)=m_{\curvearrowleft}(\emptyset)$, more precisely its transformation $\log \frac{1}{1-\kappa}$.

After appearing of counter-examples, very important W. Liu's approach [14] appeared in 2006 followed by a series of other approaches and their modifications. W. Liu suggested a two-dimensional conflict measure composed from $m_{\circledast}(\emptyset)$ and $\operatorname{DifBet} P_{m_{j}}^{m_{i}}$ - a maximal difference of $\operatorname{BetP}(A)$ for $\mathrm{BF} \mathrm{Bel}_{i}, \mathrm{Bel}_{j}$ over the focal elements $A \subset \Omega$ (thus a kind of a distance of BFs); as it was shown, neither $m_{\circledast}(\emptyset)$ nor any distance of BFs may be used as a convenient measure of conflict of BFs.

Further, we have to mention two axiomatic approaches to conflict of BFs by Destercke \& Burger [11] and by Martin [15]. In 2010, Daniel distinguished internal conflict inside an individual BF from the conflict between them [4] and defined three new approaches to conflict; the most prospective of them - plausibility conflict - was further elaborated in [6, 10]. Finally, Daniel's conflict based on non-conflicting parts of BFs was introduced in [7]. This last mentioned measure motivated our current research of hidden conflict and its special case - hidden auto-conflict of BFs [9].

Among the other approaches, we can mention e.g. Burger's geometric approach [1].

\section{Hidden Conflict}

\subsection{An Introductory Example}

Let us assume two simple consistent belief functions $B e l^{\prime}$ and $B e l^{\prime \prime}$ on $\Omega_{3}=\left\{\omega_{1}, \omega_{2}, \omega_{3}\right\}$ given by the bbas $m^{\prime}\left(\left\{\omega_{1}, \omega_{2}\right\}\right)=0.6, m^{\prime}\left(\left\{\omega_{1}, \omega_{3}\right\}\right)=0.4$, and $m^{\prime \prime}\left(\left\{\omega_{2}, \omega_{3}\right\}\right)=1.0$. Then $\left(m^{\prime} @ m^{\prime \prime}\right)(\emptyset)=0$ what seems - and it is usually considered - to be a nonconflictness of $m^{\prime}$ and $m^{\prime \prime}$, but there is positive conflict based on non-conflicting parts $\operatorname{Conf}\left(\mathrm{Bel}^{\prime}, \mathrm{Bel}^{\prime \prime}\right)=$ $\left(m_{0}^{\prime} @ m_{0}^{\prime \prime}\right)(\emptyset)=0.4>0$. (This holds true despite of Theorem 4 from [7] which should be revised in future).

We can easily verify this: the only focal element of $m^{\prime \prime}$ has a non-empty intersection with both focal elements of $m^{\prime}$, thus $\sum_{(X \cap Y)=\emptyset} m^{\prime}(X) m^{\prime \prime}(Y)$ is an empty sum; Bel is consonant, thus $B e l_{0}^{\prime \prime}=B e l^{\prime \prime}, m_{0}^{\prime \prime}=$ $m^{\prime \prime}, P l^{\prime}\left(\left\{\omega_{1}\right\}\right)=1, P l^{\prime}\left(\left\{\omega_{2}\right\}\right)=0.6, P l^{\prime}\left(\left\{\omega_{3}\right\}\right)=$ 0.4 , thus $m_{0}^{\prime}\left(\left\{\omega_{1}\right\}\right)=0.4, m_{0}^{\prime}\left(\left\{\omega_{1}, \omega_{2}\right\}\right)=0.2$, $m_{0}^{\prime}\left(\left\{\omega_{1}, \omega_{2}, \omega_{3}\right\}\right)=0.4$, hence $\operatorname{Conf}\left(B e l^{\prime}, B_{e l}^{\prime \prime}\right)=$ $\left(m_{0}^{\prime} @ m_{0}^{\prime \prime}\right)(\emptyset)=m_{0}^{\prime}\left(\left\{\omega_{1}\right\}\right) m_{0}^{\prime \prime}\left(\left\{\omega_{2}, \omega_{3}\right\}\right)=0.4 \cdot 1=0.4$. 


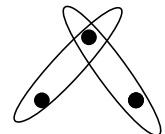

(1)

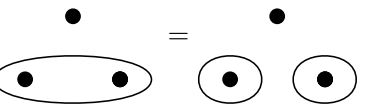

Figure 1: Introductory Example: focal elements of $m^{\prime}, m^{\prime \prime}$, and of $m^{\prime} @ m^{\prime \prime}$.

Table 1: Hidden conflict in the Introductory Example

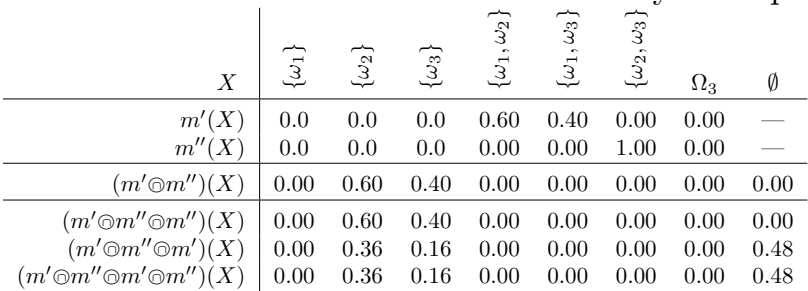

\subsection{Interpretation of the Example - Observation of a Hidden Conflict}

The following questions arise: Does $\left(m^{\prime} @ m^{\prime \prime}\right)(\emptyset)=$ 0 really represent non-conflictness of respective BFs as it is usually assumed? Is the definition of conflict based on non-conflicting parts correct? Are $m^{\prime}$ and $m^{\prime \prime}$ conflicting? What does $\left(m^{\prime} @ m^{\prime \prime}\right)(\emptyset)=0$ mean?

Suppose that $\mathrm{Bel}^{\prime}$ and $\mathrm{Bel}^{\prime \prime}$ are non-conflicting now. Thus their combination should be also nonconflicting with both of them. Does this hold for BFs from our example? This holds true when we combine $m^{\prime} @ m^{\prime \prime}$ with $m^{\prime \prime}$ one more time (assuming two instances of $m^{\prime \prime}$ comming from two independent belief sources). It follows from the idempotency of categorical $m^{\prime \prime}: m^{\prime} @ m^{\prime \prime} @ m^{\prime \prime}=m^{\prime} @ m^{\prime \prime}$ and therefore $\left(m^{\prime} @ m^{\prime \prime} @ m^{\prime \prime}\right)(\emptyset)=0$ again. On the other hand, we obtain positive $\left(m^{\prime} @ m^{\prime \prime} @ m^{\prime}\right)(\emptyset)=$ $\left(m^{\prime} @ m^{\prime} @ m^{\prime \prime}\right)(\emptyset)=0.48$ (assuming $m^{\prime}$ comming from two independent belief sources again). See Table 1 and Figure 2. When $m^{\prime \prime}$ and $m^{\prime}$ are combined once, then we observe $m_{\circledast}(\emptyset)=0$. When combining $m^{\prime \prime}$ with $m^{\prime}$ twice then $m_{\circledast}(\emptyset)=0.48$. We observe some kind of a hidden conflict. Moreover, both individual BFs are consistent. I.e. there are no internal conflicts. Thus our hidden conflict is hidden conflict between the BFs and we have an argument for correctness of positive value of $\operatorname{Conf}\left(B e l^{\prime}, B e l^{\prime \prime}\right)$.

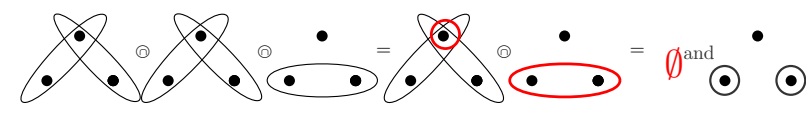

Figure 2: Arising of a hidden conflict between BFs in the Introductory Example: focal elements of $m^{\prime}, m^{\prime}, m^{\prime \prime}-m^{\prime} @ m^{\prime}, m^{\prime \prime}$ and of $\left(m^{\prime} @ m^{\prime}\right) @ m^{\prime \prime}$.

What is a decisional interpretation of our BFs? Contours, i.e. plausibilities of singletons are $P l^{\prime}=$ $(1.0,0.6,0.4)$ and $P l^{\prime \prime}=(0.0,1.0,1.0)$, we obtain
$P l_{-} P^{\prime}=(0.5,0.3,0.2)$ and $P l_{-} P^{\prime \prime}=(0.0,0.5,0.5)$ by normalization; thus at $B e l^{\prime}, \omega_{1}$ is significantly preferred, whereas at $B e l^{\prime \prime}$, one of $\omega_{2}, \omega_{3}$; this is also an argument for a positive value of mutual conflict of the BFs. Considering Smets' pignistic probability we obtain $\operatorname{Bet} P^{\prime}=(0.5,0.3,0.2)$ and $\operatorname{Bet} P^{\prime \prime}=$ $(0.0,0.5,0.5)$, just the same values as in the case when normalized plausibility of singletons (normalized contour) is used for decision. Both the, in general different, probabilistic approximations $B e t P$ and $P l_{-} P$ give the highest value to different singletons for $B e l^{\prime}$ and $B e l^{\prime \prime}$. Thus the argument for mutual conflictness of the BFs is strengthened and we obtain the same pair of incompatible decisions based on the BFs in both frequent decisional approaches: using either normalized contour (which is compatible with the conjunctive combination of BFs) or pignistic probability (designed for betting).

Hence $\left(m^{\prime} @ m^{\prime \prime}\right)(\emptyset)$ does not mean non-conflictness of the BFs. It means simple or partial compatibility of their focal elements only.

\subsection{Objections Against Our Interpretation}

There are several objections to our interpretation of the introductory example:

- in case of a combination of two identical belief functions some idempotent rule of combination should be used. Yes, this would be right for belief functions coming from two dependent belief sources. But this is not true for two or more numerically same BFs coming from two or more independent belief sources.

- The result is not surprising, because conflict is increasing when combining more belief functions. This is definitely not true:

- to be correct it should be stated 'nondecreasing' instead of increasing.

- more precisely, a conflict is non-decreasing when more conflicting BFs are combined. When truly non-conflicting BFs are combined, any positive conflict can never arise there; e.g. $\left(\bigcirc_{1}^{k} \operatorname{Bel}_{1} @ \bigcirc_{1}^{k} \operatorname{Bel}_{2}\right)(\emptyset)=0$ for any $k>0$ and Bel $_{i}$ on $\Omega_{3}$ given by $m_{1}\left(\left\{\omega_{1}\right\}\right)=0.3, m_{1}\left(\left\{\omega_{1}, \omega_{2}\right\}\right)=0.2$, $m_{1}\left(\left\{\omega_{1}, \omega_{3}\right\}\right)=0.1, m_{1}\left(\left\{\omega_{1}, \omega_{2}, \omega_{3}\right\}\right)=0.4$, $m_{2}\left(\left\{\omega_{1}, \omega_{3}\right\}\right)=0.7, m_{2}\left(\left\{\omega_{1}, \omega_{2}, \omega_{3}\right\}\right)=0.3$.

- The result is rather unsurprising. Because one can see clearly that the hidden conflict occurs when the first combination results in disjoint focal sets. Yes, in the very simple Introductory Example, this may by unsurprising for someone; but there are no disjoint sets after the first 
combination in the following Little Angel example. Moreover, this should be surprising for all who accept the following assumption / axiom: BFs Bel' and Bel" are non-conflicting whenever $\left(m^{\prime} @ m^{\prime \prime}\right)(\emptyset)=0$, e. g. $[1,11,14,15]$ and the previous Daniel's publications, e. g. $[6,7,10]$.

- It is obvious that a combination results in a conflict if a Bayesian BF $\left(m^{\prime} @ m^{\prime \prime}\right.$ in the Introductory Example) is combined with any other BF. Yes, this is true in the very simple introductory example, but not in a general example, see e. g. the following Little Angel example again.

Analyzing these objections we can see, why it was not easy to observe the hidden conflicts: in simple cases, the observation seems to be obvious, thus not interesting, in more general examples this seems to be really hidden.

\subsection{Definition of Hidden Conflict}

Definition 1 Let us suppose two BFs $\mathrm{Bel}^{\prime}, \mathrm{Bel}^{\prime \prime}$ defined by bbas $m^{\prime}, m^{\prime \prime}$, such that $\left(m^{\prime} @ m^{\prime \prime}\right)(\emptyset)=0$. If there further holds $\left(m^{\prime} @ m^{\prime \prime} @ m^{\prime}\right)(\emptyset)>0$ or $\left(m^{\prime} \bigcirc m^{\prime \prime} @ m^{\prime \prime}\right)(\emptyset)>0$ we say that there is a hidden conflict of the BFs.

Observation $1 A$ condition $\left(m^{\prime} @ m^{\prime \prime} @ m^{\prime}\right)(\emptyset)>0$ or $\left(m^{\prime} @ m^{\prime \prime} @ m^{\prime \prime}\right)(\emptyset)>0$ from Definition 1 is equivalent to the following condition $\left(m^{\prime} @ m^{\prime \prime} @ m^{\prime} @ m^{\prime \prime}\right)(\emptyset)>0$.

We have to note that a hidden conflict is quite a new phenomenon, qualitatively different from the ideas of all previous Daniel's works on conflict of belief functions and also different from the other referred approaches. Till now, it was supposed that $m_{\circlearrowleft}(\emptyset)$ includes both, the conflict between BFs and also internal conflicts of individual BFs. Thus the conflict between BFs was supposed to be less or equal to $m_{\circledast}(\emptyset)$. Here, we deal with a situation of a positive conflict between BFs while $m_{\circledast}(\emptyset)=0$.

We have already observed that $m_{\odot}(\emptyset)=0$ does not mean full non-conflictness of BFs and that the condition $\left(m^{\prime} @ m^{\prime \prime} @ m^{\prime} @ m^{\prime \prime}\right)(\emptyset)>0$ together with $\left(m^{\prime} @ m^{\prime \prime}\right)(\emptyset)=0$ defines hidden conflict. What about the condition $\left(m^{\prime} @ m^{\prime \prime} @ m^{\prime} @ m^{\prime \prime}\right)(\emptyset)=0$ ? Is this condition sufficient for full non-conflictness of BFs Bel and $B e l^{\prime \prime}$ ? May some conflict be still hidden there?

The zero version of the condition seems to imply nonconflictness on $\Omega_{3}$, the frame of discernment of the Introductory Example. To solve the question in general, we have to consider a larger frame of discernment.

\subsection{Little Angel Example}

For $\Omega_{5}$ one can find the following Little Angel Example, see Table 2. Similarly to Introductory Example, we have two consistent BFs Bel ${ }^{i}$ and $\mathrm{Bel}^{i i}$ with disjoint sets of max-plausibility elements while zero condition $\left(m^{i} @ m^{i i}\right)(\emptyset)=0$ holds true.

Table 2: Hidden Conflict in the Little Angel Example

\begin{tabular}{r|ccccc}
$X$ & $\begin{array}{c}A= \\
\left\{\omega_{1}, \omega_{2}, \omega_{5}\right\}\end{array}$ & $\begin{array}{c}B= \\
\left\{\omega_{1}, \omega_{2}, \omega_{3}, \omega_{4}\right\}\end{array}$ & $\begin{array}{c}C= \\
\left\{\omega_{1}, \omega_{3}, \omega_{4}, \omega_{5}\right\}\end{array}$ & $\begin{array}{c}X= \\
\left\{\omega_{2}, \omega_{3}, \omega_{4}, \omega_{5}\right\}\end{array}$ & $\emptyset$ \\
\hline$m^{i}(X)$ & 0.10 & 0.30 & 0.60 & 0.00 & - \\
$m^{i i}(X)$ & 0.00 & 0.00 & 0.00 & 1.00 & - \\
\hline
\end{tabular}

\begin{tabular}{r|ccccccc}
$X$ & $A \cap X$ & $B \cap X$ & $C \cap X$ & $\begin{array}{c}A \cap B \\
\cap X\end{array}$ & $\begin{array}{c}A \cap C \\
\cap X\end{array}$ & $\begin{array}{c}B \cap C \\
\cap X\end{array}$ & $\emptyset$ \\
\hline$\left(m^{i} \odot m^{i i}\right)(X)$ & 0.10 & 0.30 & 0.60 & 0.00 & 0.00 & 0.00 & 0.00 \\
\hline$\left(m^{i} \odot m^{i i} \odot m^{i i}\right)(X)$ & 0.10 & 0.30 & 0.60 & 0.00 & 0.00 & 0.00 & 0.00 \\
$\left(m^{i} \odot m^{i} \odot m^{i i}\right)(X)$ & 0.01 & 0.09 & 0.36 & 0.06 & 0.12 & 0.36 & 0.00 \\
$\left(m^{i} \odot m^{i} \odot m^{i i} \odot m^{i i}\right)(X)$ & 0.01 & 0.09 & 0.36 & 0.06 & 0.12 & 0.36 & 0.00 \\
\hline$\left(m^{i} \odot m^{i i} \odot m^{i i} \odot m^{i i}\right)(X)$ & 0.010 & 0.090 & 0.360 & 0.060 & 0.120 & 0.360 & 0.000 \\
$\left(m^{i} \odot m^{i} \odot m^{i} \odot m^{i i}\right)(X)$ & 0.001 & 0.027 & 0.216 & 0.036 & 0.126 & 0.486 & 0.108 \\
$m^{i} \odot m^{i} \odot m^{i} \odot m^{i i} \odot m^{i i} \odot m^{i i}(X)$ & 0.001 & 0.027 & 0.216 & 0.036 & 0.126 & 0.486 & 0.108
\end{tabular}

In addition to Introductory Example, $\left(m^{i} @ m^{i i} @ m^{i} @ m^{i i}\right)(\emptyset)=0 \quad($ see Table 2) while Conf $\left(\mathrm{Bel}^{i}, \mathrm{Bel}^{i i}\right)=0.1$ is positive again. Positiveness of the Conf value can be easily seen from the fact that sets of max-plausibility elements are disjoint for $P l^{i}$ and $P l^{i i}$. Numerically, we have again $B e l_{0}^{i i}=B e l^{i i}$, and $P l_{-} P^{i}=\left(\frac{10}{39}, \frac{4}{39}, \frac{9}{39}, \frac{9}{39}, \frac{7}{39}\right)$. We obtain $m_{0}^{i}\left(\left\{\omega_{1}\right\}\right)=0.1, m_{0}^{i}\left(\left\{\omega_{1}, \omega_{3}, \omega_{4}\right\}\right)=$ $0.2, m_{0}^{i}\left(\left\{\omega_{1}, \omega_{3}, \omega_{4}, \omega_{5}\right\}\right)=0.3, m_{0}^{i}\left(\left\{\Omega_{5}\right\}\right)=0.4$, and Conf $\left(\right.$ Bel $^{i}$, Bel $\left.^{i i}\right)=m_{0}^{i}\left(\left\{\omega_{1}\right\}\right) m^{i i}(X)=0.1$. Analogous arguments hold true for the positive Conf and hidden conflict again (of the 2nd degree this time). Bet $^{i}=(0.2583,0.1083,0.2250,0.2250,0.1833)$ which is not numerically the same as $P l_{-} P^{i}$, but both prefer $\omega_{1}$, whereas $\operatorname{Bet} P^{i i}=P l_{-} P^{i i}=$ $(0.00,0.25,0.25,0.25,0.25)$.

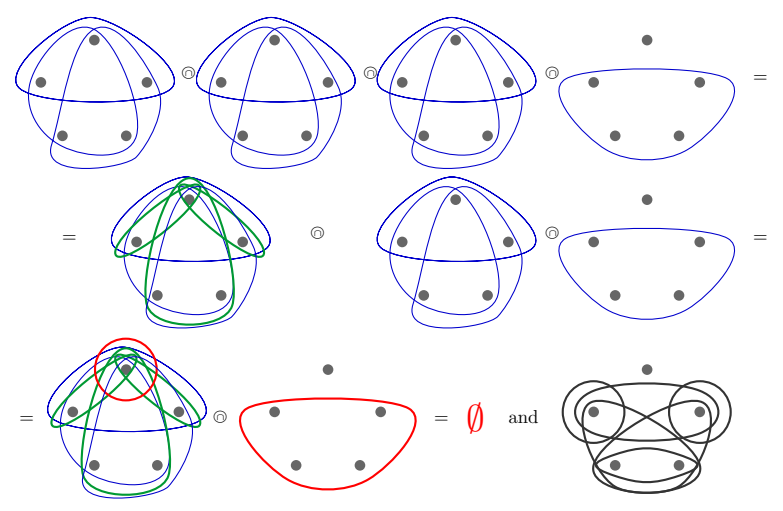

Figure 3: Arrising of a hidden conflict between BFs in the Little Angel Example. Focal elements of $m^{i}, m^{i i}$, $m^{i} @ m^{i}, m^{i} @ m^{i} @ m^{i}$ and of $\left(m^{i} @ m^{i} @ m^{i}\right) @ m^{i i}$. Red-colored focal elements are those responsible for creation of the empty-set in the last step.

For the existence of a hidden conflict, it is the structure of focal elements that is important — not their belief 
masses. Belief masses are important for the size of a conflict. In general, we can take $m^{i}(A)=a, m^{i}(B)=$ $b, m^{i}(C)=c$, for $A, B, C$ defined in Table 2 , and for any $a, b, c>0$, such that $a+b+c=1$, we obtain $m(\emptyset)=6 a b c$ as a hidden conflict of the 2nd degree (in our numeric case there is $6 a b c=6 \cdot 0.1 \cdot 0.3 \cdot 0.6=0.108)$. For graphical presentation of the Little Angel Example see Figure 3.

Degrees of hidden conflict, its maximal value, and the issue of full non-conflictness will be analyzed in the following section.

\section{Degrees of Hidden Conflict and Full Non-conflictness}

When analyzing examples from the previous section, we have observed different degrees of hidden conflict. We can formalize it in the next definition.

Definition 2 Assume two BFs $\mathrm{Bel}^{i}$, Bel ${ }^{i i}$ defined by bbas $m^{i}, m^{i i}$, such that for some $k>0$ $\left(\bigcirc_{j=1}^{k}\left(m^{i} @ m^{i i}\right)\right)(\emptyset)=0$. If there further holds $\left(\bigcirc_{j=1}^{k+1}\left(m^{i} @ m^{i i}\right)\right)(\emptyset)>0$ we say that there is a conflict of BFs Bel and $\mathrm{Bel}^{i i}$ hidden in the $k$-th degree.

Analogously to particular degrees of hidden conflict, there are degrees of non-conflictness. Particular degrees of non-conflictness are not very important. However, there is an important question whether there is some hidden conflict or not, i.e. whether or not the $\mathrm{BF}$ s in question are fully non-conflicting.

Definition 3 We say that BFs Bel ${ }^{i}$ and $\mathrm{Bel}^{i i}$ are fully non-conflicting if $\left(m^{i} \circlearrowleft m^{i i}\right)(\emptyset)=0$ and, further, if there is no hidden conflict of any degree. I.e. if $\left(\bigcirc_{j=1}^{k}\left(m^{i} @ m^{i i}\right)\right)(\emptyset)=0$ for any $k \geq 0$.

Thus there is a question how many times we have to combine $\left(m^{i} @ m^{i i}\right)$, i.e., for which $k$ value of $\left(\bigcirc_{j=1}^{k}\left(m^{i} @ m^{i i}\right)\right)(\emptyset)$ shows whether there is some hidden conflict of the BFs Bel and Bel ${ }^{i i}$ or not. For answers to this question see corollaries of the following two theorems.

Theorem 4 (maximal degree of hidden conflict) For any non-vacuous BFs Bel ${ }^{i}$ and $\mathrm{Bel}^{i i}$ defined by $m^{i}$ and $m^{i i}$ on any frame $\Omega_{n}$ it holds that

$\left(\bigcirc_{j=1}^{n-1}\left(m^{i} @ m^{i i}\right)\right)(\emptyset)=0 \quad$ iff $\left(\bigcirc_{j=1}^{k}\left(m^{i} \odot m^{i i}\right)\right)(\emptyset)=0$ for any $k>n-2$.

Corollary $\mathbf{5}$ Hidden conflict of any non-vacuous BFs on any $\Omega_{n}$ has always degree less or equal to $n-2$; i.e., the condition

$$
\left(\bigcirc_{j=1}^{n-1}\left(m^{i} @ m^{i i}\right)\right)(\emptyset)=0
$$

always means full the non-conflictness of any BFs Bel ${ }^{i}$ and $\mathrm{Bel}^{i i}$ on any $\Omega_{n}$.

Let us present an example of such a highly hidden conflict now.

Example 6 Example of hidden conflict of the $n$ - 2-th degree: Let us suppose n-element frame of discernment $\Omega_{n}=\left\{\omega_{1}, \omega_{2}, \ldots, \omega_{n}\right\} . \quad B e l^{i}$ and $B e l^{i i}$ are given by $m^{i}\left(\left\{\omega_{1}, \omega_{2}, \ldots, \omega_{n-1}\right\}\right)=$ $\frac{1}{n-1}, \quad m^{i}\left(\left\{\omega_{1}, \omega_{2}, \ldots, \omega_{n-2}, \omega_{n}\right\}\right)=\frac{1}{n-1}$, $m^{i}\left(\left\{\omega_{1}, \omega_{2}, \ldots, \omega_{n-3}, \omega_{n-1}, \omega_{n}\right\}\right)=\frac{1}{n-1}, \ldots m^{i}\left(\left\{\omega_{1}\right.\right.$, $\left.\left.\omega_{3}, \omega_{4}, \ldots, \omega_{n}\right\}\right)=\frac{1}{n-1}, m^{i i}\left(\left\{\omega_{2}, \omega_{3}, \ldots, \omega_{n}\right\}\right)=\frac{1}{1} . A t$ $m^{i} @ m^{i}(n-2)$-element focal elements appear, at $m^{i} @ m^{i} @ m^{i}(n-3)$-element focal elements appear, at $\bigcirc_{j=1}^{k} m^{i}(n-k)$-element focal elements appear, at $\bigcirc_{j=1}^{n-2} m^{i}$ 2-element focal elements appear, all these focal elements have non-empty intersections with the only focal element of $m^{i i}$, and finally at $\bigcirc_{j=1}^{n-1} m^{i}$ singleton focal element $\left\{\omega_{1}\right\}$ appears which has empty intersection with the only focal element of $m^{i i}\left\{\omega_{2}, \omega_{3}, \ldots, \omega_{n}\right\}$.

What does $m^{i}$ express? It gives a big support to all elements of the frame, to the entire frame $\Omega_{n}$ and even greater support to $\omega_{1}$ which is included in all focal elements; $\omega_{1}$ is preferred and, moreover, it has plausibility 1 . We can modify $m^{i}$ and express this more easily: $\bar{m}^{i}\left(\Omega_{n}\right)=\frac{n-1}{n}, \bar{m}^{i}\left(\left\{\omega_{1}\right\}\right)=\frac{1}{n}$, or more generally, $\widetilde{m}^{i}\left(\Omega_{n}\right)=1-a, \widetilde{m}^{i}\left(\left\{\omega_{1}\right\}\right)=a$ for some $0<a<1$. We can easily see evident conflict corresponding to positive $\bar{m}(\emptyset)=\left(\bar{m}^{i} \circlearrowleft m^{i i}\right)(\emptyset)=\frac{1}{n}, \widetilde{m}(\emptyset)=a$ for these modifications of $m^{i}$. Hence either hidden conflict of the $(n-2)$-th degree of $m^{i}$ and $m^{i i}$ or positive $\operatorname{Conf}\left(m^{i}, m^{i i}\right)=\operatorname{Conf}\left(\bar{m}^{i}, m^{i i}\right)=\frac{1}{n}$ should not be very surprising.

We have to note that the Introductory Example is a special instance of Example 6 for $n=3$.

Theorem 7 (i) Any non-vacuous BFs Bel ${ }^{i}, \mathrm{Bel}^{i i}$ have a conflict hidden at most in $(c-1)$-th degree where $c=\min \left(c^{i}, c^{i i}\right)+\operatorname{sgn}\left(\left|c^{i}-c^{i i}\right|\right)$. where $c^{i}, c^{i i}$ are maximal cardinalities of focal elements of $\mathrm{Bel}^{i}, \mathrm{Bel}^{i i}$ different from $\Omega$. In the other words

$\left(\bigcirc_{j=1}^{c}\left(m^{i} @ m^{i i}\right)\right)(\emptyset)=0 \quad$ iff $\left(\bigcirc_{j=1}^{k}\left(m^{i} @ m^{i i}\right)\right)(\emptyset)=0$ for any $k \geq c=\min \left(c^{i}, c^{i i}\right)+\operatorname{sgn}\left(\left|c^{i}-c^{i i}\right|\right)$.

(ii) There are no hidden conflicts of any non-vacuous BFs on any two-element frame $\Omega_{2}$.

(iii) There are no hidden conflicts of any non-vacuous quasi-Bayesian BFs on any frame $\Omega_{n}$.

(iv) For a BF Bel and a quasi-Bayesian BF Bel $\mathrm{B}^{i i}$ there is a hidden conflict of (at most) the first degree; if it appears then it is an internal conflict of $\mathrm{Bel}^{i i}$ in fact. 


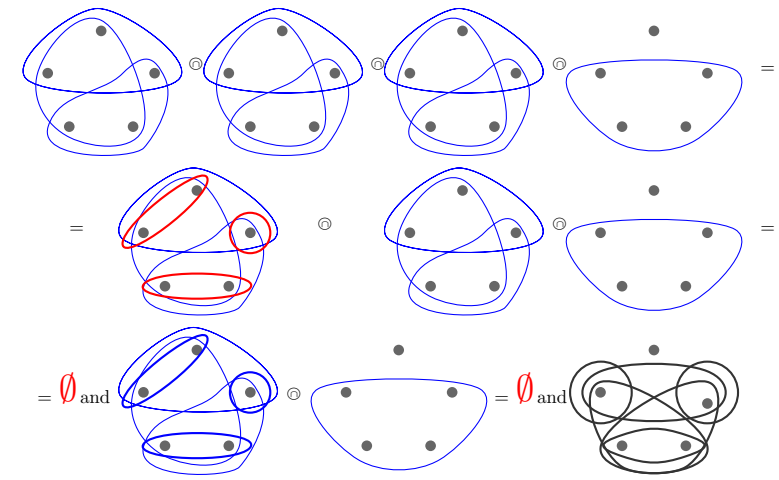

Figure 4: Arrising of an internal hidden conflict of $\mathrm{BF} \mathrm{Bel}^{i i i}$ in the Little Angel Modified Example. Focal elements of $m^{i i i}, m^{i i}, m^{i i i} @ m^{i i i}, m^{i i i} @ m^{i i i} @ m^{i i i}$, and of $\left(m^{i i i} @ m^{i i i} @ m^{i i i}\right) @ m^{i i}$. Red-colored focal elements are those responsible for creation of the emptyset in the next step.

Corollary 8 (i) Assume two non-vacuous BFs $\mathrm{Bel}^{i}, \mathrm{Bel}^{i i}$ on $\Omega_{n}$. The zero value of the expression $\left(\bigcirc_{j=1}^{c}\left(m^{i} @ m^{i i}\right)\right)(\emptyset)$, i.e., the condition

$$
\left(\bigcirc_{j=1}^{c}\left(m^{i} @ m^{i i}\right)\right)(\emptyset)=0
$$

means full non-conflictness of the BFs for $c=$ $\min \left(c^{i}, c^{i i}\right)+\operatorname{sgn}\left(\left|c^{i}-c^{i i}\right|\right)$, where $c^{i}, c^{i i}$ are maximal cardinalities of focal elements of $\mathrm{Bel}^{i}, \mathrm{Bel}^{i i}$ different from $\Omega_{n}$.

(ii) For any two non-vacuous quasi Bayesian BFs $\mathrm{Bel}^{i}, \mathrm{Bel}^{i i}$ on any frame of discernment $\Omega_{n}$ the condition $\left(m^{i} \odot m^{i i}\right)(\emptyset)=0$ always means full nonconflictness of the BFs.

(iii) For any BF Bel $l^{i}$ and any quasi-Bayesian BF $B e l^{i i}$ the condition $\left(\bigcirc_{j=1}^{2}\left(m^{i} @ m^{i i}\right)\right)(\emptyset)=0$ always means full non-conflictness of the BFs.

\section{Internal Hidden Conflict}

\section{Little Angel Modification}

Let us take $m^{i i i}$ instead of $m^{i}$, such that $m^{i i i}(A)=m^{i}(A), \quad m^{i i i}(C)=m^{i}(C)$, and $m^{i i i}(D)=m^{i i i}\left(\left\{\omega_{2}, \omega_{3}, \omega_{4}\right\}\right)=0.30$ instead of $m^{i}(B)$. There is $\left(m^{i i} @ m^{i i} @ m^{i i i} @ m^{i i i}\right)(\emptyset)=0$, but $\left(m^{i i} \bigcirc m^{i i i} \bigcirc m^{i i i} \bigcirc m^{i i i}\right)(\emptyset) \quad>\quad 0, \quad$ even $\left(m^{i i i} @ m^{i i i} @ m^{i i i}\right)(\emptyset)>0$, i.e. $\left(\bigcirc_{1}^{3} m^{i i i}\right)(\emptyset)>0$, for detail see [8] and Figure 4.

We observe a conflict of the belief functions hidden in the 2nd degree again. Nevertheless, the situation of focal elements is different now: the only focal element $X$ of $m^{i i}=\bigcirc_{1}^{3} m^{i i}$ has non-empty intersection with any focal element of $\cap_{1}^{3} m^{i i i}$, but $\left(\cap_{1}^{3} m^{i i i}\right)(\emptyset)>0$ now. Thus this is not a hidden conflict between $m^{i i}$ and $m^{i i i}$, but an internal hidden conflict of $m^{i i i}$.

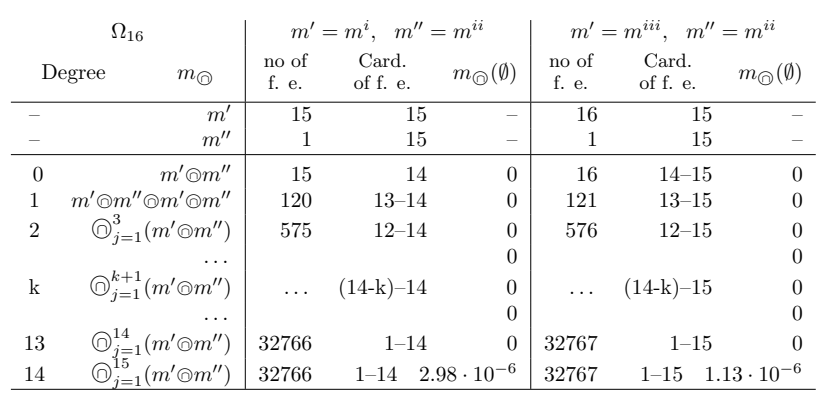

Table 3: Hidden conflict between BFs $\mathrm{Bel}^{i}$ and $\mathrm{Bel}^{i i}$ from Example 6 and internal hidden conflict of $B e l^{i i}$ and $\mathrm{Bel}^{i i i}$ from Example 9, both on $\Omega_{16}$.

Computation of $\bigcirc_{1}^{k}\left(m^{\prime} @ m^{\prime \prime}\right)(\emptyset)$ and internal hidden conflict have a relation to Martin's auto-conflict [16, 15], thus we can speak about hidden auto-conflict here. See [9] for more details.

In general, we can observe internal hidden conflict when at least one of BFs in hidden conflict is not consistent. Let us present an example with highly hidden internal hidden conflict of the $(n-2)$-th degree on a general frame of discernment now.

Example 9 Let us consider the following modification of Example 6 on $\Omega_{n}$. Instead of $m^{i}$ we take $m^{i i i}$ having all focal elements of cardinality $n-1$, such that $m^{i i i}\left(\Omega_{n} \backslash\{\omega\}\right)=\frac{1}{n}$ for any $\omega \in \Omega_{n} ; m^{i i}$ same as in Example 6. $m^{i i i}$ is not consistent; $P l^{i i i}(\{\omega\})=\frac{n-1}{n}$ for any $\omega \in \Omega_{n}$. We observe hidden conflict of the $(n-2)$-th degree again. Because of same plausibilities of all singletons $m_{0}^{\prime \prime \prime}\left(\Omega_{n}\right)=1$ and Conf $\left(m^{i i i}, m^{i i}\right)=0$ now.

There is a positive hidden conflict of BFs $\mathrm{Bel}^{i i i}$ and $B e l^{i i}$, but zero conflict between them. We say that there is an internal hidden conflict. This corresponds to non-consistency of $\mathrm{BF} \mathrm{Bel}^{i i i}$; $\mathrm{Bel}^{i i}$ is consistent thus there is an internal hidden conflict of BF Bel $l^{i i i}$ in this case.

A numeric example was computed on $\Omega_{16}$, see Table 3 for a comparison of focal elements and $m_{\odot}(\emptyset)$ values of Examples 6 and 9 . For simplicity, same bbms $m^{i}(X)=$ $\frac{1}{15}$ and $m^{i i}(X)=\frac{1}{16}$ were used there.

\section{Computational Complexity and Computations of Examples}

Based on Definition 2 and Theorem 4, the complexity of computation of the degree of hidden conflict of two BFs Bel ${ }^{i}$ and $\mathrm{Bel}^{i i}$ is - on a general $\Omega_{n}$ $O(n)$ of $@$ operations. In the case of checking existence of a hidden conflict of the BFs we obtain the complexity $O\left(\log _{2}(n)\right)$ of $@$ operations utilizing a sim- 
plification of computation based on $\bigcirc_{j=1}^{2 k}\left(m^{i} @ m^{i i}\right)=$ $\bigcirc_{j=1}^{k}\left(m^{i} @ m^{i i}\right) @ \bigcirc_{j=1}^{k}\left(m^{i} @ m^{i i}\right)$. Note that the complexity of (a) operation depends on the number and structure of focal elements.

During our analysis of hidden conflicts a series of example computations was performed on frames of discernment of cardinality from 5 to 16 . A number of focal elements rapidly grows up to $|\mathcal{P}(\Omega)|=2^{|\Omega|}-1$ when conjunctive combination $@$ is repeated, see e.g. 32766 and 32767 focal elements in the presented Examples 6 and 9 at Table 3. Because the degree of the hidden conflict and existence of the hidden conflict depends on the number and the structure of focal elements not on their bbms, we have used same bbms for all focal elements of a BF in our computations on frames of cardinality greater than 10 .

All our experiments were performed in Language $\mathrm{R}$ [17] using R Studio [18]. We are currently developing an $\mathrm{R}$ package for dealing with belief functions on various frames of discernment. It is based on a relational database approach - nicely implemented in $\mathrm{R}$, in package called data.table [12].

\section{Several Important Remarks}

We have to underline that hidden conflict of belief functions is not a new measure of conflict. It just improves/extends the classic measure defining the conflict by $m(\emptyset)$ in situations where $m(\emptyset)=0$; it distinguishes fully non-conflicting BFs from those with a positive hidden conflict. This notion serves for deeper understanding of conflictness / non-conflictness, it enables to point out the conflict also in situations where conflicts had not been expected, in situations where $m_{\odot}(\emptyset)=0$; hence to point out and to help to understand the conflicts which are hidden due to $m_{\curvearrowleft}(\emptyset)=0$.

Particular numeric values of a hidden conflict have no reasonable interpretation so far. For now, we are interested whether the value is zero (i.e. no conflict) or not.

Repeated applications of the conjunctive combination (1) of a BF with itself is used here to simulate situations where different independent believers have numerically the same bbm. Thus this has nothing to do with idempotent belief combination (where of course no conflict between two BFs is possible).

There is brand new idea of hidden conflicts in [9] and in this contribution. The brand new interpretation of $m(\emptyset)$ distinguishing fully non-conflicting BFs from those with hidden conflict. The assumption of nonconflictness when $m_{\odot}(\emptyset)=0$ was relaxed, due to observation of a qualitatively new phenomena - observation of hidden conflict even in the cases where $m_{\circledast}(\emptyset)=0$. Both these studies want to point out the existence of hidden (auto-)conflicts in situations where no conflict was expected until now. Thus the definitions of hidden conflict and hidden auto-conflict[9] are not anything against the previous Daniel's research and results on conflict of belief functions e.g. $[4,6,7]$. Of course, some parts of the previous approaches should be updated to be fully consistent with the newly presented results on hidden conflicts and auto-conflicts.

Our study was motivated by the investigation of conflict Conf of BFs based on their non-conflicting parts [7], thus we were interested in independent BFs when a hidden conflict was observed. But we have to note that conflictness / non-conflictness of BFs has nothing to do with dependence/independence of the BFs. Repeated computation of several (up to $\mathrm{n}$ ) numerically identical BFs, when looking for hidden conflict is just a technical tool for computation of $m(\emptyset)$ or more precisely say for computation of $\kappa=\sum_{X \cap Y=\emptyset} m_{j}(X) m_{j}(Y)$. We are not interested in entire result of repeated application of $($, , we are interested only in $m(\emptyset)$ or more precisely say in $\kappa=\sum_{X_{1} \cap X_{2} \cap \ldots \cap X_{k}=\emptyset} m_{j}\left(X_{1}\right)$ $m_{j}\left(X_{2}\right) \ldots m_{j}\left(X_{K}\right)$. Thus our computation has nothing to do with any idempotent combination of BFs. And we can compute hidden conflict using $\bigcirc_{1}^{k}($ or $\kappa)$ in the same way for both dependent and independent BFs. It is either not necessary to include any independence assumption to our Definitions 1 and 2 .

\section{Summary and Conclusion}

Hidden conflicts of belief functions in situations where mutual intersections of any focal element of one BF with all focal element of the other BF are non-empty has been presented and analyzed. There may be a positive conflict in situations, where sums of conflicting belief masses are empty, i.e. in situations which have been usually considered to be non-conflicting till now.

Several levels — degrees of hidden conflict were observed, a maximal degree of hidden conflicts dependent on the size of the corresponding frame of discernment was found. A variety of hidden conflicts of degrees 1 $-(n-2)$ was described for an $n$-element frame of discernment. A necessary and sufficient condition for full non-conflictness of BFs in dependence on maximal cardinality of their focal elements has been specified and computational aspects analyzed. Analogously to the evident conflicts, internal hidden conflicts are distinguished from the hidden conflicts between BFs.

This qualitatively new phenomenon of conflicts of BFs moves us to a better understanding of the nature of conflicts of belief functions in general and brings a 
challenge to elaborate and update existing approaches to conflicts of BFs.

This may consequently serve as a basis for a better combination of conflicting belief functions and better interpretation of the results of belief combination whenever conflicting belief functions appear in real applications.

\section{Acknowledgement}

This work was supported by grant GAČR 19-04579S.

\section{References}

[1] T. Burger, Geometric views on conflicting mass functions: From distances to angles, International Journal of Approximate Reasoning 70 (2016) 3650 .

[2] B. R. Cobb, P. P. Shenoy, On the plausibility transformation method for translating belief function models to probability models, International journal of approximate reasoning 41 (3) (2006) $314-330$.

[3] M. Daniel, Probabilistic transformations of belief functions, in: L. Godo (Ed.), ECSQARU 2005, Vol. 3571 of LNCS (LNAI), Springer, Heidelberg, 2005, pp. 539-551.

[4] M. Daniel, Conflicts within and between belief functions, in: E. Hüllermeier, R. Kruse, F. Hoffmann (Eds.), IPMU 2010, Vol. 6178 of LNCS, Springer, Berlin, Heidelberg, 2010, pp. 696-705.

[5] M. Daniel, Non-conflicting and conflicting parts of belief functions, in: 7th International symposium on Imprecise Probability: Theories and Applications (ISIPTA 2011), SIPTA, Innsbruck, 2011, pp. 149-158.

[6] M. Daniel, Properties of plausibility conflict of belief functions, in: L. Rutkowski (Ed.), ICAISC 2013, Vol. 7894 of LNCS, Springer, Heidelberg, 2013, pp. 235-246.

[7] M. Daniel, Conflict between belief functions: A new measure based on their non-conflicting parts, in: F. Cuzzolin (Ed.), BELIEF 2014, Vol. 8764 of LNCS, Springer, Cham, 2014, pp. 321-330.

[8] M. Daniel, V. Kratochvíl, Hidden conflicts and degrees of non-conflictness of belief functions (full text in preparation), http://www.cs.cas.cz/ $\sim$ milan/for_download/hidden_conflict_ext.pdf.

[9] M. Daniel, V. Kratochvíl, Hidden auto-conflict in the theory of belief functions, in: Proceedings of the 20th Czech-Japan Seminar on Data Analysis and Decision Making under Uncertainty, 2017, pp. $34-45$.

[10] M. Daniel, J. Ma, Conflicts of belief functions: Continuity and frame resizement, in: U. Straccia, A. Cal (Eds.), SUM 2014, Vol. 8720 of LNCS, Springer, Cham, 2014, pp. 106-119.

[11] S. Destercke, T. Burger, Toward an axiomatic definition of conflict between belief functions, IEEE transactions on cybernetics 43 (2) (2013) 585-596.

[12] M. Dowle, A. Srinivasan, data.table: Extension of 'data.frame', r package version 1.10 .0 (2016). URL https://CRAN.R-project.org/package =data.table

[13] E. Lefèvre, Z. Elouedi, How to preserve the conflict as an alarm in the combination of belief functions?, Decision Support Systems 56 (2013) 326333 .

[14] W. Liu, Analyzing the degree of conflict among belief functions, Artificial Intelligence 170 (11) (2006) 909-924.

[15] A. Martin, About conflict in the theory of belief functions, in: Belief Functions: Theory and Applications, Springer, Heidelberg, 2012, pp. 161168.

[16] A. Martin, A.-L. Jousselme, C. Osswald, Conflict measure for the discounting operation on belief functions, in: Information Fusion, 2008 11th International Conference on, IEEE, 2008, pp. 1-8.

[17] R Core Team, R: A Language and Environment for Statistical Computing, R Foundation for Statistical Computing, Vienna, Austria (2016).

URL https://www.R-project.org/

[18] RStudio Team, RStudio: Integrated Development Environment for R, RStudio, Inc., Boston, MA (2015).

URL http://www.rstudio.com/

[19] J. Schubert, The internal conflict of a belief function, in: Belief Functions: Theory and Applications, Springer, Heidelberg, 2012, pp. 169-177.

[20] G. Shafer, A mathematical theory of evidence, Vol. 1, Princeton university press Princeton, 1976.

[21] P. Smets, Decision making in the tbm: the necessity of the pignistic transformation, International Journal of Approximate Reasoning 38 (2) (2005) 133-147.

[22] P. Smets, Analyzing the combination of conflicting belief functions, Information fusion 8 (4) (2007) 387-412. 